\title{
Christenverfolgungen im Römischen Reich. Elemente eines imperialen Niedergangs
}

\section{Florian Ambach}

Kerngebiet: Alte Geschichte

eingereicht bei: Mag. Simone Pittl

eingereicht im: SoSe 2017

Rubrik: PS-Arbeit

\section{Abstract \\ Persecution of Christians in the Roman Empire: Elements of an imperial decline}

The following seminar paper aims to examine how the persecution of Christians in the early centuries of the Christian era relates to the decline of the Roman Empire. It gives an overview of the period from Nero's persecutions in $64 \mathrm{AD}$ to the legalization of Christianity in $313 \mathrm{AD}$, or the end of the civil wars between Constantine and his rivals Maxentius, Maximinus Daia and Licinius around 324 AD respectively. It puts a special emphasis on the essential characteristics of what is called an "empire".

\section{Einleitung}

Die Diskussion über die Christenverfolgungen im Römischen Reich war und ist immer noch ein kontrovers diskutiertes Forschungsfeld, das bis heute nichts an politischem Sprengstoff eingebüßt hat. In einer Zeit, in der das Christentum in der arabischen Welt massiv vom sogenannten „Islamischen Staat" zurückgedrängt und existenziell bedroht wird, werden in der Forschung wieder stärker die Ursprünge und Hintergründe der frühchristlichen Christenverfolgung ins Auge gefasst und eine Brücke zu den Christen- 
verfolgungen im 20. und 21. Jahrhundert ${ }^{1}$ geschlagen, wobei ein eindeutiger Schwerpunkt auf Verfolgungen in islamischen Ländern gesetzt wird. ${ }^{2}$

Die Geschichtsforschung hat sich bis weit ins 20. Jahrhundert hinein mit den Christ_innen solidarisiert und deren Verfolgung meistens als eine systematische und eine von vornherein sorgfältig geplante betrachtet. ${ }^{3}$ Das Ziel dieser Arbeit ist zu beweisen, dass dies nicht der Fall war und dass die klassische Historiographie in der Beurteilung der einzelnen Kaiser oft nur die Kategorien Gut und Böse kannte, ohne genauere Differenzierungen vorzunehmen.

Anhand der Beschäftigung mit den Christenverfolgungen können typisch imperiale Merkmale Roms ausgemacht und analysiert werden. Der polyethnische und in unserem Fall besonders der multireligiöse Charakter, den Imperien notwendigerweise annehmen müssen, um einen dauerhaften inneren Frieden, eine Pax, gewährleisten zu können, fällt während des Niedergangs von Imperien in sich zusammen. Es zeigen sich in solchen Fällen die Grenzen der Aufnahmekapazität: Das Bestreben eines Reiches, immer mehr ethnische, kulturelle und religiöse Gruppen integrieren zu wollen, führt in vielen Fällen zu einem imperial overstretch, der das gesamte Gebilde zum Platzen bringt. ${ }^{4}$ Gestaute Konfliktpotenziale entladen sich plötzlich und in der Folge werden Minderheiten oft brutal verfolgt und auszulöschen versucht.

Da die Beweisführung ausschließlich entlang solcher Theorien gewisse Probleme mit sich bringen kann, werden in der Arbeit immer wieder Primärquellen herangezogen. An mancher Stelle wird dies aufgrund des Umfangs der Arbeit nicht möglich sein. Deshalb werden in solchen Fällen Historiker_innen zitiert, die sich eingehender mit der Kritik und Auswertung spezifischen Quellenmaterials auseinandergesetzt haben.

Eine weitere Schwierigkeit ist die lange in der Geschichtsforschung verbreitete Annahme einer systematisch geregelten Rechtslage der Christ_innen, ${ }^{5}$ etwa eines allgemeinen Verbots des Christentums. Die Realität war etwas komplexer: Es gab verschiedenste Rechtstraditionen im Imperium, die von Provinz zu Provinz voneinander abwichen und oftmals im Widerspruch zu den kaiserlichen Verordnungen standen. Darüber hinaus gab es oft unabhängig agierende Statthalter sowie spontan aufgeflammte Ausschreitungen den Christ_innen gegenüber.

1 Zusammenfassung über die weltweiten Christenverfolgungen im 20. Jahrhundert: Andrea Riccardi, Salz der Erde, Licht der Welt. Glaubenszeugnis und Christenverfolgungen im 20. Jahrhundert, Freiburg im Breisgau 2002.

2 Daniel Williams, Die Christen des Nahen Ostens - verfolgt und vergessen (Migration 3), Münster 2016; Ursula Spuler-Stegemann (Hrsg.), Feindbild Christentum im Islam. Eine Bestandsaufnahme (Herder Spektrum 5437), Freiburg im Breisgau 2004.

3 Jacques Moreau, Die Christenverfolgung im Römischen Reich (Aus der Welt der Religion 2), Berlin-New York ${ }^{2} 1971$, S. $11-13$

4 Paul Kennedy, The Rise and Fall of the Great Powers. Economic Change and Military Conflict from 1500 to 2000, New York 1987, S. 514-515; Michael Gehler/Robert Rollinger, Imperien und Reiche in der Weltgeschichte. Epochenübergreifende und globalhistorische Vergleiche, in: Michael Gehler/Robert Rollinger (Hrsg.), Imperien und Reiche in der Weltgeschichte. Epochenübergreifende und globalhistorische Vergleiche 1, Wiesbaden 2014, S. 1-28, hier S. 5-6.

5 Olaf Sild, Das altchristliche Martyrium in Berücksichtigung der rechtlichen Grundlage der Christenverfolgung, Dorpat 1920, S. 43-51. 
In der Arbeit werden zunächst allgemeine religionspolitische Merkmale des Imperium Romanum thematisiert, bevor die ersten Christenverfolgungen einschließlich jener unter Nero anlässlich des Brandes von Rom im Jahr 64 n. Chr. beschrieben werden. Im darauffolgenden Kapitel geht es um die punktuell auftretenden Verfolgungen während der Herrschaft der Flavischen und der Antoninischen Dynastie. Bevor es in der Chronologie mit den Severern und den Soldatenkaisern weitergeht, ist ein Kapitel eingeschoben, in dem auf die innerkirchlichen Streitfragen eingegangen wird. Den von Decius initiierten Christenverfolgungen wird ein eigenes Kapitel eingeräumt, die einzelnen Verfolgungen von Valerian und Aurelian hingegen nur grob skizziert, ehe im letzten Kapitel die Rolle der Christ_innen in der Tetrarchie und im Bürgerkrieg genauer untersucht wird.

\section{Religionspolitik von der republikanischen Zeit bis Nero}

Die Tatsache, dass Rom eine große Zahl seiner Götter aus dem Pantheon der Griech_innen übernommen hat und diese sich mit bereits vorhandenen italischen Gottheiten vermischen, ist richtungsweisend für die frühe Religionspolitik. Antonie Wlosok hat gezeigt, dass der Gottesdienst der Römer vor allem Mittel der Propaganda und der Legitimation war, also eine Art integrativer Staatskult. Gegen Ende der republikanischen Epoche fanden neue Kulte aus dem Osten Eingang in die römische Götterwelt. Im Rahmen des Bacchanalienprozesses um 186 v. Chr. wurden geheime Gesellschaften verboten, doch nicht aufgrund des Glaubens, sondern weil die Versammlung mehrerer Gleichgesinnter und die ausschweifenden Praktiken, die gegen die mores maiorum verstießen, für den Staat und die Bevölkerung zunehmend als Bedrohung empfunden wurden. Ähnlich suspekt waren die aus Griechenland importierten philosophischen Lehren der Pythagoreer, Epikureer, Skeptizisten und besonders jene der Stoiker, die durch ihren abstrakten Gottesbegriff die "Volksgötter" anzweifelten und ihnen nur „Volkserzieherischen Wert" beimaßen. ${ }^{6}$ Die neuen Kulte und Lehren schufen die Möglichkeit von persönlicher Frömmigkeit. Weil sie das Individuum in gewissem Maße immun gegen die öffentliche und meinungsbildende Staatsreligion machten, stellten sie eine bis dahin ungekannte innenpolitische Bedrohung dar. Im Jahre 59 v. Chr. wurde das Isis-Heiligtum auf dem Kapitol in Rom zerstört. Der Bürgerkrieg zwischen Octavian und Antonius wurde bereits von den Zeitgenoss_innen als Glaubenskrieg der italischen gegen die orientalischen Götter stilisiert.

Während Tertullian und Plinius der Ältere Kaiser Nero (54-68) als den ersten Verfolger ausmachen ${ }^{8}$, weist Augustinus darauf hin, dass Christ_innen bereits vorher verfolgt

6 Antonie Wlosok, Rom und die Christen. Zur Auseinandersetzung zwischen Christentum und römischem Staat, in: Der Altsprachliche Unterricht 13 (1970), Beiheft 1, S. 62-63.

7 Cassius Dio, Dio's Roman History, Bd. 5, Griechisch-englisch, übersetzt von Earnest Cary, herausgegeben von Thomas Ethelbert Page/William Henry Denham Rouse (Loeb Classical Library 82), London-New York 1917, S. 489490.

8 Tertullian, Tertullians ausgewählte Schriften, Bd. 2 (Bibliothek der Kirchenväter, 1. Reihe, Bd. 24), übersetzt von Heinrich Kellner, herausgegeben und kommentiert von Carl Andresen, München 2011², S. 325-326; Karl Julius Sillig (Hrsg.), C. Plini Secundi Naturalis Historiae, Bd. 3, Hamburg-Gotha 1853, S. 68. 
wurden. ${ }^{9}$ Diese Verfolgungen, denen Jesus und dann auch Stephanus und Jakobus zum Opfer fielen, gingen allerdings von den Jüdinnen und Juden aus, die die römischen Behörden auf die Sekte aufmerksam gemacht hatten.

Die Schuldfrage für den Brand Roms im Jahre 64 n. Chr. ist bis heute ungelöst und wird es vermutlich auch bleiben. Während eine Gruppe von Historiker_innen ${ }^{10}$ die Schuld Nero zuschreibt, der sich durch seine Abwesenheit von der Stadt verdächtig gemacht hatte und durch den Brand sein lang geplantes Renovierungsprojekt endlich in Angriff nehmen konnte, werden von einer anderen Gruppe ${ }^{11}$ die Christ_innen verdächtigt, da ausgerechnet ihre Wohnviertel weitgehend verschont geblieben waren. Die moderne Forschung ${ }^{12}$ geht hingegen von einem zufällig ausgebrochenen Feuer aus, das durch die in diesem Sommer besonders starke Hitze und durch den kräftigen Wind verursacht wurde. Die mangelnde Wasserversorgung schloss ein schnelles Bekämpfen des Brandes aus. ${ }^{13}$ Für die Katastrophe wurden dann die verhassten Christ_innen verantwortlich gemacht. Wahrscheinlich trug die Gattin Neros, Poppaea Sabina, entscheidend dazu bei, die Christ_innen als Schuldige darzustellen, da sie Kontakt zu jüdischen Kreisen unterhielt, die die Christ_innen als Sekte ansahen. In der Forschung ist umstritten, ob es einen Prozess gab oder nicht. Ein sogenanntes Institutum Neronianum, ein kaiserliches Gesetz, das die Verfolgung der Christ_innen regelte, wird angenommen. ${ }^{14}$ Jedenfalls soll Nero die Verfolgung wie ein Schauspiel gestaltet haben, um die Rachsucht der Bevölkerung zu befriedigen. Den Christ_innen wurde nachgesagt, nachts geheime Messen abzuhalten, Inzest zu treiben, Kinder zu töten und sie anschließend zu verspeisen. Diese Gerüchte nährten den Hass der Römer_innen auf sie. Entsprechend entwürdigend waren die Verurteilungen: Den Christ_innen sollen Tierfelle umgelegt worden sein, manche wurden den Hunden vorgeworfen, andere gekreuzigt und wieder andere verbrannt, sodass sie die Straßen nachts beleuchteten. Tacitus hebt hervor, dass sie weniger aufgrund der Brandstiftung als wegen ihres odium humani generis (Hass gegen das Menschengeschlecht) verurteilt wurden. ${ }^{15}$ Diese Anspielung lässt durchscheinen, dass Anhänger_innen des Christentums der römischen Bevölkerung durch ihre Zurückgezogenheit von der Öffentlichkeit und Fixierung auf das Jenseits suspekt vorgekommen sein mussten.

9 Augustinus, Vom Gottesstaat (De civitate Dei). Vollständige Ausgabe in einem Band, übersetzt von Wilhelm Thimme, eingeleitet und kommentiert von Carl Andresen, München 2011², S. 507-508.

10 Cassius Dio, Dio's Roman History, Bd. 5, S. 71; Sueton, Leben und Taten der römischen Kaiser (Kaiserviten). Über berühmte Männer, herausgegeben und übersetzt von Werner Krenkel und Adolf Stahr, Köln 201322, S. 325-326; Plinius der Ältere in der Edition Sillig, C. Plini Secundi Naturalis Historiae, S. 68.

11 Anton August Draeger (Hrsg.), Die Annalen des Tacitus. Schulausgabe, Bd. 2, Leipzig 1869, S. 185.

12 Gregory Neil Daugherty, The cohortes vigilium and the Great Fire of 64 AD, in: Classical Journal 87 (1991), S. 229-240.

13 Hans Dieter Stöver, Christenverfolgung im Römischen Reich. Ihre Hintergründe und Folgen, Düsseldorf-Wien 1982, S. 15-17.

14 Jan Willem Philip Borleffs, Institutum Neronianum, in: Richard Klein (Hrsg.), Das frühe Christentum im römischen Staat (Wege der Forschung 267), Darmstadt 1971, S. 217-235; Jacques Zeiller, Institutum Neronianum. Hirngespinst oder Wirklichkeit?, in: Richard Klein (Hrsg.), Das frühe Christentum im römischen Staat (Wege der Forschung 267), Darmstadt 1971, S. 236-243.

15 Draeger, Die Annalen des Tacitus, S. 189. 


\section{Von Domitian bis Commodus: conquirendi non sunt ${ }^{16}$}

Nach dem Tod Neros im Jahr 68 kam es zunächst zu keinen weiteren Verfolgungen. Erst unter Domitian (81-96) wurde im Jahre 95 der ehemalige Konsul Glabrio, der möglicherweise mit dem Christentum sympathisierte, als molitor rerum novarum („Ausbrüter neuer Dinge“) hingerichtet. ${ }^{17}$ Dieser Fall zeigt, dass das Christentum langsam in die elitäre Senatorenschicht einsickerte - für den Staat eine alarmierende Bedrohung, gegen die man vorgehen musste. Domitian begann eine Judenverfolgung, die auch Christ_ innen traf. Im ersten Clemensbrief an die Korinther wurde eine Verfolgung angedeutet. ${ }^{18}$ Nachdem Domitian einen Enkel des Bruders Jesu - also einen Angehörigen des königlichen Geschlechts Davids - verhört hatte und feststellen konnte, dass dieser keine politischen Absichten hatte, sondern betonte, das Reich Christi sei himmlisch, ließ er die Verfolgung einstellen.

Nach Domitians Tod im Jahr 96 genossen die Christ_innen eine relative Friedenszeit wenn sie verfolgt wurden, dann nur aufgrund einzelner Statthalter, die in vielen Fällen, insbesondere nach Naturkatastrophen, Ernteausfällen und Seuchen, dem Druck der Bevölkerung nachgaben. Damit lenkten sie den Unmut von sich selbst ab und schufen gewissermaßen ein Ventil für die Unzufriedenheit der Bevölkerung.

Trajan (98-117) betraute seinen Vertrauensmann Plinius den Jüngeren zwischen 111 und 113 mit dem Amt eines „kaiserlichen Statthalters mit allen Rechten eines corrector, curator und kaiserlichen procurator ${ }^{\prime \prime 19}$ in der Provinz Pontus und Bithynien. Dieser wandte sich mit einem Schreiben an den Kaiser, in dem er ihn um genaue Anweisungen bat, wie er mit den Christ_innen verfahren solle und zu klären, ob ein Unterschied zu machen sei bezüglich Alter und Geschlecht. Zudem musste geklärt werden, ob nur bekennende Christ_innen oder auch jene, die von Christus abgeschworen haben, zu verurteilen seien und ob das nomen ipsum (Christsein als solches) oder erst etwaige flagitia cohaerentia (mit dem Christentum in Verbindung stehende Verbrechen) ${ }^{20}$ zu bestrafen seien. Plinius ging so vor, dass nur diejenigen, die sich nach dreimaliger Befragung unter Androhung der Todesstrafe nicht dem Kaiser opferten und Christus schmähten, hingerichtet wurden. ${ }^{21}$ Trajans Reskript billigte dieses relativ milde Vorgehen und betonte das conquirendinon sunt ("gefahndet werden soll nach ihnen nicht“). ${ }^{22}$ Dies kann ein Hinweis darauf sein, dass die "Seuche dieses bösen Aberglaubens" 23 bereits so weit verbreitet war, dass an eine totale Vernichtung nicht mehr zu denken war. Nur wenn die Christ_innen den Behörden freiwillig in die Hände liefen und sich dem

16 Plinius der Jüngere, Des Plinius Cäcilius Secundus Werke, Bd. 4, Lateinisch-deutsch, herausgegeben und übersetzt von Johann Adam Schäfer, Wien-Triest 1814, S. 236.

17 Sueton, Leben und Taten der römischen Kaiser, S. 418-419.

18 Moreau, Die Christenverfolgung, S. 40.

19 Rudolf Freudenberger, Das Verhalten der römischen Behörden gegen die Christen im 2. Jahrhundert dargestellt am Brief des Plinius an Trajan und den Reskripten Trajans und Hadrians (Münchener Beiträge zur Papyrusforschung und antiken Rechtsgeschichte 52), München 1967, S. 19.

20 Plinius der Jüngere, Des Plinius Cäcilius Secundus Werke, S. 228.

21 Ebd.

22 Ebd., S. 236

23 Ebd., S. 235. 
Opfer verweigerten, wurden sie hingerichtet. Darüber hinaus unterstrich Trajan, dass man sich nicht auf anonym eingereichte Denunziationen stützen solle. ${ }^{24}$

Hadrian (117-138) behielt diese Richtlinien bei. In einem Reskript an den Prokonsul der Provinz Asia, Gaius Minucius Fundanus, bekräftigte er wie sein Vorgänger nachdrücklich, dass Anklagen auf einfache Beschuldigungen hin fallen zu lassen seien. ${ }^{25}$

Für die Regierungszeit Mark Aurels ist ein rapider Anstieg von Martyrien nachweisbar. Ausgerechnet der "Philosophenkaiser", der sich um einen ausgewogenen Regierungsstil bemühte, wurde in eine Zeit der allgemeinen Krise und des Niedergangs hineingeboren. Hans Dieter Stöver sieht die ganze Tragik des Mark Aurel darin, dass er seine staatsphilosophischen Theorien unter dem Druck der allgemeinen Unzufriedenheit über Bord werfen musste und sich gezwungen sah, durch härteres Durchgreifen dem chaotischen Zustand des Imperiums entgegenzutreten - wenngleich es seinen ethischen Ansichten widersprach, Menschen ihres Glaubens wegen zu verfolgen. Stöver zählt in den 19 Jahren seiner Regierung 17 Kriege. Dazu kamen Verwüstung ganzer Landstriche, Ernteausfälle, Hungerkatastrophen, finanzielle Krisen, Überschwemmungen und die Pest, an der Mark Aurel schließlich selbst zugrunde ging. ${ }^{26}$

Am besten lässt sich diese aufgeheizte Atmosphäre in dem Massaker von Lyon im Jahre 177 nachweisen. Dort versammelten sich die Vertreter_innen der gallischen Provinzen für die Feiern des Kaiserkultes. Plötzlich entlud sich die Anspannung und Christ_innen wurden von der Bevölkerung angegriffen und durch die gesamte Stadt hindurch verfolgt. Viele Christ_innen wurden niedergemetzelt - zum Teil auch ohne ein gerichtliches Verfahren. ${ }^{27}$

Die bekanntesten Märtyrer_innen unter Mark Aurel waren Bischof Polykarp, Publius von Athen, der Apologet Justin von Rom und Blandina. ${ }^{28}$ In diese Zeit fallen auch erste Märtyrerberichte, von denen später noch die Rede sein wird. Trotz dieser hohen Zahl von Märtyrer_innen wurde Mark Aurel von der christlichen Historiographie nicht explizit als Verfolger abgestempelt. Tertullian sprach ihm sogar eine Schutzfunktion zu, die er den Christ_innen gegenüber aus seiner Dankbarkeit für das „Regenwunder" ${ }^{\prime 29}$ im Markomannenkrieg angenommen habe. ${ }^{30}$

24 Plinius, Werke, S. 236.

25 Freudenberger, Das Verhalten der römischen Behörden, S. 218.

26 Stöver, Christenverfolgung im Römischen Reich, S. 97-115.

27 Ebd., S. 78-96.

28 Eusebius, Ausgewählte Schriften Bd. II: Kirchengeschichte, Lateinisch-deutsch, herausgegeben und übersetzt von Philipp Haeuser (Bibliothek der Kirchenväter, 2. Reihe, Bd. 1), München 1932, S. 207-221.

29 Das sogenannte "Regenwunder" ereignete sich wahrscheinlich am 11. Juni 172 im Rahmen des Ersten Markomannenkrieges, in dem Kaiser Mark Aurel die in das Römische Reich eindringenden Germanenstämme bekämpfte. Die römischen Truppen litten unter der Hitze und der vorherrschenden Wasserknappheit. Erst durch ein plötzlich eintretendes Gewitter konnten sie ihren Durst stillen und zu einem erfolgreichen Gegenschlag gegen den Stamm der Quaden ausholen. Von christlichen Geschichtsschreibern wurde das Ereignis auf das Gebet eines christlichen Soldaten zurückgeführt, der den Beistand Gottes erwirkt habe. Hansjörg Ubl, Das Regenwunder im Quadenland, in: Alfred Stirnemann (Hrsg.), 1000 Jahre Ostarrîchi. Seine christliche Vorgeschichte. Mission und Glaube im Austausch zwischen Orient und Okzident (Pro Oriente 19), Innsbruck-Wien 1997, S. 16-22.

Tertullian, Tertullians ausgewählte Schriften, S. 54-55. 
Auch Commodus (180-192) befahl keine Verfolgung, doch es kam zu vielen Tumulten, bei denen unter anderem die dreizehn Märtyrer von Scili (17. Juli 180) und Apollonius von Rom (184 oder 185) starben. Die Verzweiflung und Überforderung der römischen Behörden lassen sich am besten im Ausspruch des Prokonsuls von Asien, Arrius Antonius, fassen: „Wenn ihr Unseligen schon sterben wollt, habt ihr denn keine Stricke, gibt es keine Abgründe?"31

\section{Exkurs: Innerkirchliche Auseinandersetzungen und Heiligenverehrung}

Für die Christ_innen waren die Verfolgungen eine harte Probe, die auch entscheidend dazu beitrug, die christliche Lehre zu verändern und gegenüber anderen Deutungen abzugrenzen. Die Geschichtsforschung ist lange von einem einigen Christentum ausgegangen, das sich bereits klar definiert hatte. Dabei wurde allerdings außer Acht gelassen, dass die Christ_innen nicht nur das Römische Reich als Feind von außen hatten, sondern dass der Formierungsprozess der Religion voll im Gange war: Auf mehreren lokalen Konzilien wurde kontrovers über dogmatische, soteriologische, christologische und ekklesiologische Fragen diskutiert. Die dort getroffenen Entscheidungen hatten meist nur regionale Gültigkeit, sodass sich eine Vielzahl an verschiedenen Glaubensrichtungen herausbildete. Diese spalteten sich von der Kirche, zu deren Mittelpunkt Rom wurde, ab und gingen fortan getrennte Wege. Aus diesem Grund scheint es unerlässlich, einen historischen Querschnitt zwischenzuschieben, der teilweise schon in die Zeit des Decius und des Diokletian vorgreift, um diese Verfolgungen besser nachvollziehen zu können.

Eine zentrale Frage, die sich an der Christenverfolgung entzündete, war, wie man mit den lapsi („Abgefallene“) umgehen sollte. Im Grunde machten es die römischen Behörden den Christ_innen leicht, dem Todesurteil zu entkommen, wie aus dem bereits behandelten Briefwechsel zwischen Plinius und Trajan hervorgeht. Eine rein formale Entsagung von Christus und die Opferung an den Kaiser reichten schon, um unbehelligt freigelassen zu werden. ${ }^{32}$ Doch innerhalb der Kirche führte das Problem der lapsi, die infolge der immer größer angelegten Verfolgungen häufiger wurden, zu einem Streit, das Christentum eine Zeit lang zu entzweien drohte. Während der gemäßigte Flügel die Abgefallenen wieder als Teil der Gemeinde akzeptieren wollte, sprach sich der radikalere gegen eine Wiederaufnahme aus. Cyprian von Karthago sprach sich in seiner Schrift "De lapsis" für die Reintegration aus, doch dies nur um den Preis einer glaubhaften und ernst gemeinten Buße. Der radikalere Flügel der Montanisten, deren Wortführer Tertullian war, forderte von den Christ_innen, in den Verfolgungen standhaft zu bleiben und bis zum Äußersten zu gehen. ${ }^{33}$ In „De pallio“ spricht er vom Ablegen seiner Toga, der repräsentativen Kleidung eines römischen Bürgers, zugunsten des Palliums

31 Tertullian, Schriften, S. 272

32 Moreau, Die Christenverfolgung, S. 86

33 Susanne Hausammann, Verfolgungs- und Wendezeit der Kirche. Gemeindeleben in der Zeit der Christenverfolgungen und Konstantinische Wende (Alte Kirche. Zur Geschichte und Theologie in den ersten vier Jahrhunderten 2), Neukirchen-Vluyn 2001, S. 75-76. 


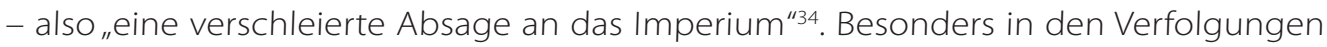
unter Decius und Diokletian zeigte sich ein regelrechter Märtyrerwunsch. So wurden etwa von den 43 Opfern der diokletianischen Verfolgung in Palästina allein 21 wegen ihres provozierenden Verhaltens gegenüber den Behörden oder durch Selbstanzeige überführt. ${ }^{35}$ Cyprian hingegen warnte vor übermütigem Handeln, ${ }^{36}$ da er wusste, dass im "Martyrium Polycarpi", dem ersten Märtyrerbericht, von einem phrygischen Christen die Rede war, der die Aufmerksamkeit der Verwaltung auf sich lenkte, dann unter der Folter aber nachgab. ${ }^{37}$ Kurz vor der decischen Verfolgung im Jahre 257 beklagte Origenes die mangelnde Opferbereitschaft und Verkalkung, in die das Christentum abgesunken sei. ${ }^{38}$

Das eben erwähnte Genre der Märtyrerberichte war für die Christ_innen von äußerster Wichtigkeit. Darin wurde minutiös beschrieben, welchen Qualen sie standhalten mussten. Im Grunde entwickelte sich dadurch der ausgeprägte Heiligenkult, der das Christentum auch in den nachfolgenden Jahrhunderten prägte. ${ }^{39}$ Er stand dafür, dass auch Angehörige der untersten Schichten durch ihren Glauben die Märtyrerkrone und damit Ruhm und Glanz erlangen konnten - also ein wichtiges integratives Element im Christentum. Jährlich an ihrem Todestag wurde der Märtyrer gedacht und ihr Beispiel schweißte die Zurückgebliebenen zusammen und rief sie auf, Ähnliches zu erdulden.

Eine weitere Textform, zu der die Christ_innen griffen, war die Apologie, eine Art gerichtliche Verteidigungsrede. Bekannte Namen von Apologeten sind Justin, Melito von Sardes, Minucius Felix, Apollinaris von Ravenna, Irenäus von Lyon, Clemens von Alexandria, Origenes, Cyprian, Papst Miltiades, Eusebius und Augustinus. Exemplarisch herausgegriffen werden soll hier der "Octavius" des Minucius Felix, eines nordafrikanischen Rechtsanwaltes, der zum Christentum konvertiert war. Die Apologie wurde gegen Ende des zweiten oder Anfang des dritten Jahrhunderts verfasst. Darin verteidigt Octavius das Christentum gegen seinen Freund Caecilius, der folgenden Vorwurf bringt: latebrosa et lucifugax natio, in publicum muta, in angulis garrula ("ein duckmäuseriges und lichtscheues Volk, stumm in der Öffentlichkeit, nur in den Winkeln gesprächig"). ${ }^{40}$ Er sieht das Christentum also als eine Bedrohung an, die aus den untersten Schichten hervorwachse. Als Skeptizist verwirft er zwar aufgrund der humana mediocritas

34 Denis van Berchem, Tertullians "De pallio" und der Konflikt des Christentums mit dem Imperium Romanum, in: Richard Klein (Hrsg.), Das frühe Christentum im römischen Staat (Wege der Forschung 267), Darmstadt 1971, S. 106-128, hier S. 126.

35 Hedwig Fritzen, Methoden der diokletianischen Christenverfolgung. Nach der Schrift des Eusebius über die Märtyrer in Palästina, phil. Diss. München 1962, S. 58.

36 Cyprian, Des heiligen Kirchenvaters Caecilius Cyprianus Briefe (Bibliothek der Kirchenväter, 1. Reihe, Bd. 60), übersetzt von Julius Baer, herausgegeben von Otto Bardenhewer/Thomas Schermann/Carl Weyman, München 1928, S. 410.

37 Martyrium Polycarpi, Das Martyrium des Polykarp (Kommentar zu den Apostolischen Vätern 6), übersetzt und kommentiert von Gerd Buschmann, Göttingen 1998, S. 119-120.

38 Origenes, Acht Bücher gegen Celsus (Bibliothek der Kirchenväter, 1. Reihe, Bd. 52), herausgegeben und übersetzt von Paul Koetschau, München 1926, S. 127-128.

39 Hausammann, Verfolgungs- und Wendezeit der Kirche, S. 19-58.

40 Minucius Felix, Frühchristliche Apologeten, Bd. 2 (Bibliothek der Kirchenväter, 1. Reihe, Bd. 14), übersetzt von Alfons Müller, herausgegeben von Otto Bardenhewer/Thomas Schermann/Carl Weyman, Kempten-München 1913, S. 147. 
(„menschliche Beschränktheit“) ${ }^{41}$ die Möglichkeit der Gotteserkenntnis, spricht sich aber dennoch für die Verehrung der heidnischen Götter aus, weil es gelte, Traditionen zu pflegen.42 Octavian hält dem entgegen, dass alle Menschen - egal ob arm oder reich - Gott erkennen können. Am Ende ist Caecilius damit einverstanden und bekennt sich zum Christentum.

\section{Die Ruhe vor dem Sturm: von Septimius Severus bis Philippus Arabs}

Diese Apologie fällt genau in die Regierungszeit des Septimius Severus, der Hofämter an Christ_innen vergab und seinen Sohn und Nachfolger Caracalla von einer christlichen Amme stillen ließ. ${ }^{43}$ Ausgerechnet in einer Zeit, in der der Bestand des an all seinen Grenzen bedrohten Reiches von der Einigkeit und Zusammenarbeit aller seiner Bewohner_innen abging, wurden von christlicher Seite aus die radikalsten Parolen ausgegeben - Parolen, die zur Verweigerung des Militärdienstes ausriefen. ${ }^{44}$ Deshalb sah sich Severus gezwungen, im Jahre 202 ein Edikt zu erlassen, das den Übertritt zum Christentum unter Todesstrafe stellte. Bekannte Märtyrer_innen waren Irenäus von Lyon (um 200), Perpetua und Felicitas (7. März 203), deren Märtyerbericht stark montanistisch geprägt ist und deshalb Tertullian zugeschrieben wird, ${ }^{45}$ Potamiaina mit ihrer Mutter Marcella und der von ihnen bekehrte Soldat Basilides. ${ }^{46}$

Caracallas Erlass der "Constitutio Antoniniana“ im Jahre 212, der Verleihung des Bürgerrechts an alle freien Untertanen, wurde von Cassius Dio als fiskalpolitische Maßnahme verstanden, um das finanziell zerrüttete Reich durch vermehrte Steuereinnahmen sanieren zu können. ${ }^{47}$ Wieder andere ${ }^{48}$ wollten sie als Voraussetzung für eine allgemeine Christenverfolgung verstanden haben, da nur Vollbürger der Majestätsbeleidigung angeklagt werden konnten. ${ }^{49}$

Maximinus Thrax (235-238) begann im Jahr seines Regierungsantritts eine kurze Verfolgung gegen die Spitzen der Kirche, in der Papst Pontian und Gegenpapst Hippolyt verbannt wurden. ${ }^{50}$ Philippus Arabs (244-249) war toleranter und stand sogar mit Origenes in Briefkontakt. Von Orosius und Hieronymus wird Philippus als der erste christliche Kaiser genannt, „aber die Zeit war noch nicht reif, um sich offen zum Christentum zu bekennen." ${ }^{151}$ Er erlaubte Papst Fabian in den Jahren zwischen 244 und 249, die Reliquien seines Vorgängers Pontian nach Rom zu bringen.52

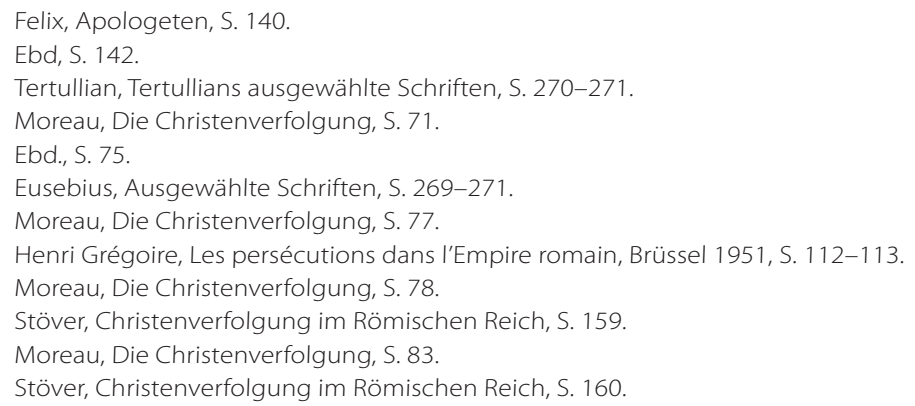




\section{Decius: das Christentum als substantielle Bedrohung?}

Zusammenfassend lässt sich sagen, dass der Großteil der Verfolgungen bis Decius (249-251) nicht von staatlicher Initiative aus geplant war, sondern eher spontan aufgrund des örtlichen Volkszorns in den Provinzen ausbrach.

Von seinem Vorgänger noch mit einem Sonderkommando an der Donau ausgestattet, wurde der Illyrer Decius 249 von seinen Truppen zum Kaiser ausgerufen. ${ }^{53}$

„Ja es scheint eine historische Regel zu sein, dass immer dann, wenn das politische, militärische oder religiöse Establishment resignieren, gefangen im Netz erstarrter Normen und phantasieloser Stagnation - ihm vom Rande her neue, unverbrauchte Kräfte zuwachsen"54

- ein klassisch imperiales Element. Unmittelbar begannen die Verfolgungen: Am 20.1.250 erlitt Papst Fabian das Martyrium und der Stuhl Petri blieb vakant. Mitte des Jahres wurde ein Edikt erlassen, das von allen Vollbürgern des Reichs eine supplicatio (Opfergabe) forderte.

Leo Koep hat das Thema der Unvereinbarkeit des römischen Kaiserkults mit der Lehre des Christentums ausgezeichnet analysiert..$^{55}$ Hier will ich seine zentralen Thesen zusammenfassen: Nach dem Tod Caesars ließ Octavian ihn sakralisieren, indem er sich selbst auf den Münzen den Titel "DIVI FILIUS" gab. Von diesem Moment an war weltliche und geistliche Macht in der Person des Kaisers verbunden. Während die alten Gottheiten immer mehr ins Hintertreffen gerieten, traten Abstraktionen (Concordia, Salus, Fortuna, Victoria, etc.) ihren Siegeszug an.

Jesus hatte dem Vorwurf, sich als König zu empfinden, nichts entgegenzusetzen allerdings verstand er sich als König eines himmlischen Reiches. Die Römer sahen in inm trotzdem eine konkrete politische Bedrohung, wobei ihm nichts ferner gewesen sein dürfte als politischer Aufruhr oder gar Usurpation. Entsprechend enthielt das über dem Kreuz befestigte Schild die Natur des Verbrechens: lesus Nazarenus Rex ludaeorum.

In seiner frühen Phase hatte das Christentum - wenigstens der gemäßigte Flügel, der den Großteil ausmachte, - keineswegs die Absicht, das Römische Reich zu stürzen. In den „Acta proconsularia Sancti Cypriani“ aus dem Jahr 258 wird Cyprian zitiert:

Huic deo nos Christiani deseruimus, hunc deprecamur diebus ac noctibus pro nobis et pro omnibus hominibus et pro incolumitate ipsorum imperatorum. ${ }^{56}$

53 Reinhard Selinger, Die Religionspolitik des Kaisers Decius. Anatomie einer Christenverfolgung (Europäische Hochschulschriften, 3/617), Berlin-Bern u. a. 1994, S. 179.

54 Stöver, Christenverfolgung im Römischen Reich, S. 167.

55 Leo Koep, Kaisertum und Christusbekenntnis im Widerspruch, in: Richard Klein (Hrsg.), Das frühe Christentum im römischen Staat (Wege der Forschung 267), Darmstadt 1971, S. 302-336.

56 Otto von Gebhardt (Hrsg.), Ausgewählte Märtyreracten und andere Urkunden aus der Verfolgungszeit der christlichen Kirche, Berlin 1902, S. 124. („Diesem Gott unterstehen wir Christ_innen und zu ihm beten wir Tag und Nacht für uns und für alle Menschen und auch für die Unversehrtheit der Kaiser.") Eigene Übersetzung. 
Dass das Christentum das Ende des Römischen Reiches besiegelt hätte, ist also zu relativieren. Im „Martyrium Polycarpi“ aus dem Jahr 155 wollte der Präfekt von Smyrna Polykarp vom Martyrium abbringen und fragte ihn deshalb: „Was ist denn schon Schlimmes

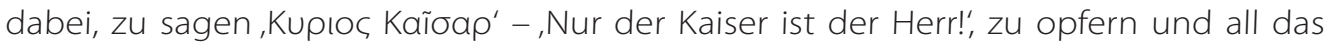
übrige tun, um sein Leben zu retten?" ${ }^{45}$

Es handelte sich also um "Zeremonien, die zwar zum religiösen Kult gehören, aber doch nur als äußerliche Bekundung bürgerlicher Loyalität, nicht religiöser Überzeugung gelten" ${ }^{\prime 58}$. Wenn der Kaiserkult nicht religiöse Elemente enthalten hätte, wären die Christ_innen dem Opferbefehl nachgekommen; so aber gingen viele von ihnen unweigerlich dem Martyrium entgegen.

Die Opfergabe lief so ab, dass jede_r römische Bürger_in dem Kaiser ein kultisch-religiöses Opfer darbringen musste, das je nach Provinz unterschiedlich durchgeführt wurde. Im Grunde war also diese Verfolgung formal nicht gegen die Christ_innen gerichtet, traf diese allerdings - und das wusste Decius - am empfindlichsten. Nach den vollzogenen Kulthandlungen erhielt jede_r Opfernde eine Opferbestätigung (libellum). Durch Folterungen wurde die Zahl der lapsi weiter in die Höhe getrieben, auch Bischöfe waren unter ihnen. Auch kam es oft vor, dass sich Christ_innen gegen Entrichtung einer Summe ein libellum erkauften, ohne das Opfer leisten zu müssen. Diesen Vorwurf richtete Gegenpapst Novatian, ein Mann montanistischer Prägung, der eine nach ihm benannte Bewegung gründete, an Papst Cornelius, der Abgefallenen gegenüber milder gestimmt war. $^{59}$

\section{Von Valerian bis Aurelian}

Die Verfolgung endete erst mit dem Tod Decius' auf dem Schlachtfeld - er war der erste Kaiser, den dieses Schicksal ereilte. Die ersten Jahre unter Valerian (253-260) verliefen durchaus friedlich. Die Staatsfinanzen waren an einem Tiefpunkt angelangt, „der Feingehalt des Silbers sank unter Decius von 50 Prozent auf 40 Prozent, unter Valerian auf 16 Prozent bis 14 Prozent und unter seinem Nachfolger Gallienus schließlich auf zwei Prozent." 60 In einem Brief des Dionysios von Alexandria, den Eusebius zitiert, wird angedeutet, dass der christenfeindliche Finanzminister Macrianus den Kaiser entscheidend zu einer neuen Verfolgung bewogen habe, ${ }^{61}$ die vor allem die Häupter der Kirche treffen sollte.

Ein erstes Edikt (August 257) forderte den Klerus zum Opfern auf und stellte ein Versammlungsverbot auf, das bei Todesstrafe die Versammlung auf den Coemeterien verbat. Da die Christ_innen keine anderen Plätze besaßen, hatte dies den Effekt einer Enteignung. ${ }^{62}$ Das zweite Edikt (August 258) ordnete die Hinrichtung der Opferverwei-

57 Martyrium Polycarpi, Das Martyrium des Polykarp, S. 161.

58 Koep, Kaisertum und Christusbekenntnis, S. 314.

59 Werner Portmann, Zu den Motiven den Diokletianischen Christenverfolgung, in: Historia 39 (1990), Heft 2, S. 212-248, hier S. 240-242.

60 Moreau, Die Christenverfolgung, S. 89

61 Stöver, Christenverfolgung im Römischen Reich, S. 178-179.

62 Hausammann, Verfolgungs- und Wendezeit der Kirche, S. 15. 
gerer an: Darunter waren auch Cyprian, Papst Sixtus II. und die 300 Märtyrer_innen von Massa Candida, die in ungelöschten Kalk geworfen wurden. ${ }^{63}$

Nachdem Valerian im Jahr 260 in die Hände des Sasanidenkönigs Shapur gefallen war und unter Folterqualen starb, ${ }^{64}$ kam sein Sohn und Mitkaiser Gallienus (253-268) an die Macht. Dieser erließ im Jahre 262 ein Toleranzedikt, das den Christ_innen ihre Kirchen und Friedhöfe zurückerstattete. ${ }^{65}$ Dadurch war der Beginn einer vierzigjährigen Friedenszeit für die Christ_innen gekennzeichnet, die kurz von Aurelian (270-275) unterbrochen wurde. Dieser wollte den Kult des Sol Invictus zur Staatsreligion durchsetzen, plante eine Verfolgung, doch starb vor der Umsetzung. ${ }^{66}$

\section{8. „Diokletianische Verfolgung“: Christ_innen als Figuren im Machtspiel der Tetrarchie}

Diokletian (284-305) wollte dem imperial overstretch entgegenwirken, indem er das Reich durch das System der Tetrarchie auf zwei Augusti aufteilte, die ihrerseits zwei Caesares ernannten, um die Kommunikation zu erleichtern. „Imperien sind [...] immer auch Räume der Kommunikation.“7 Damit verbunden war ein sakrales Herrschertum. Mit dem 19. Regierungsjahr Diokletians begann die Verfolgung: Die jüngere Forschung hat gezeigt, dass der Caesar Galerius Diokletian maßgeblich beeinflusst hat. Allerdings sei davon auszugehen, dass dieser die endgültige Zustimmung gegeben hat. ${ }^{68}$ Galerius habe „die Christenverfolgung als ein Mittel politischer Taktik ergriffen, um die Sympathien der im Wesentlichen heidnischen illyrischen Armee zu gewinnen".69

Mit dem ersten Edikt (23.2.303) wurde die Zerstörung der Kirchen, Herausgabe der heiligen Schriften und Suspendierung von Ämtern beschlossen, das zweite und dritte Edikt sollten die klerikale Schicht zum Abfall bewegen:

„man griff zu Gewalt und List. Die Gefangenen wurden zu den Altären geschleift und gegen ihren Willen gezwungen zu opfern, indem man ihnen die Hände gewaltsam mit Opfergaben [...] füllte und sie durch Martern soweit brachte, dass sie die Gaben über dem Altar fallen ließen. [...] Bei vielen unterzog man sich nicht erst der Mühe, ihnen ein Opfer abzuzwingen, sondern sie wurden

63 Stöver, Christenverfolgung im Römischen Reich, S. 180 und S. 185.

64 Laktanz, Des Lucius Caelius Firmianus Lactantius Schriften (Bibliothek der Kirchenväter, 1. Reihe, Bd. 36), übersetzt von Aloys Hartl, herausgegeben von Otto Bardenhewer/Thomas Schermann/Carl Weyman, Kempten-München 1919, S. 8-9; Robert Rollinger/Josef Wiesehöfer, Kaiser Valerian und Ilu-ibdi von Hamat. Über das Schicksal besiegter Feinde, persische Grausamkeit und die Persistenz altorientalischer Traditionen, in: Heather Baker/Kai Kaniuth/Adelheid Otto (Hrsg.), Stories of long ago. Festschrift für Michael D. Roaf (Alter Orient und Altes Testament 397), München 2012, S. 497-516.

65 Moreau, Die Christenverfolgung, S. 93.

66 Stöver, Christenverfolgung im Römischen Reich, S. 214-215.

67 Jürgen Osterhammel, Europamodelle und imperiale Kontexte, in: Journal of Modern European History 2 (2004), S. 157-182, hier S. 178-179.

68 Moreau, Die Christenverfolgung, S. 100-101.

69 Joseph Vogt, Christenverfolgung (historisch), in: Reallexikon für Antike und Christentum 2, Stuttgart 1954, Sp. 1194. 
auf die falsche Erklärung eines anderen hin, dass sie geopfert hätten, entlassen, selbst wenn sie durch lautes Rufen das Gegenteil bezeugten." ${ }^{\prime 70}$

Erst das vierte Edikt (Jänner oder Februar 304), das von Galerius während der Abwesenheit des Diokletian erlassen wurde, befahl ein reichsweites Opfer. ${ }^{71}$ Unter Maximian (286-305) gab es in Italien schon Verfolgungen, denen der hl. Sebastian, die hl. Agnes und Papst Marcellinus zum Opfer gefallen waren. ${ }^{72}$ Sein Nachfolger Maxentius (306-312) rückte von dieser Politik ab und ließ die Christ_innen nach dreijähriger Sedisvakanz einen neuen Papst, Marcellus I., wählen, der jedoch dann mit Eusebius ins Exil geschickt wurde - blutig verliefen diese Verbannungen allerdings nicht. Maxentius ließ sogar Münzen mit dem Kreuzzeichen prägen. ${ }^{73}$ In den verschiedenen Reichsteilen wurde die Verfolgung unterschiedlich intensiv betrieben: Während in Gallien und Britannien nur das erste Edikt zur Anwendung kam, wurde die Verfolgung im Osten unter Maximinus Daia über die Abdankung Diokletians (305) und sogar über das spätere Toleranzedikt des Galerius (311) hinaus betrieben. In Lorch erlitt der hl. Florian unter Galerius sein Martyrium, in Lydda oder Nikomedia der hl. Georg unter Diokletian. ${ }^{74}$ Der Terminus "diokletianische Verfolgung" ist also zu überdenken.

In einigen Fällen scheint es, dass die Statthalter die Gesetzesgrenzen übertraten: Christ_innen sollten sich in Gladiatorenkämpfen gegenseitig umbringen und Jungfrauen wurden zu sexuellen Handlungen gezwungen. Auch ist ein Fall überliefert, in dem einem Richter von dem Christen Aedesius unzureichende Rechtskenntnisse nachgewiesen wurden. ${ }^{75}$ Besonders im Osten - d. h. unter Galerius und Diokletian, später unter Maximinus Daia - erreichten die Verfolgungen ein ungekanntes Ausmaß an Brutalität. Stöver nennt das achte Buch von Eusebius' „Kirchengeschichte“ "ein einzigartiges Dokument der bestialischen Verirrungen, zu denen der Mensch fähig ist." ${ }^{\prime \prime 6}$ Die Todesstrafe des Säckens (culleus) erfreute sich größter Beliebtheit: Der Verurteilte wurde zusammen mit Tieren, wie etwa Ratten oder Skorpionen, in einen Sack eingenäht und dann ins Meer geworfen, sodass die wild gewordenen Tiere zum Angriff übergingen. Diese Art von Verurteilung entzog die Leichen der Verehrung und verhinderte dadurch den für die Christ_innen so wichtigen Heiligenkult. ${ }^{77}$

Im April 311 eilte Licinius ans Sterbebett des Galerius und konnte ihn zur Einstellung der Verfolgung bewegen - Konstantin schaltete 313 seinen Konkurrenten Maxentius in der Schlacht an der Milvischen Brücke aus und ließ sich vom Senat das Prädikat titulus primi nominis („Titel des ersten Namens“) geben - er überragte also die übrigen Mitstreiter. Im selben Jahr schickte er Licinius gegen den in Thrakien einmarschierenden Daia, der sich dem Toleranzedikt von 311 widersetzt hatte. Licinius, der den Sieg davon-

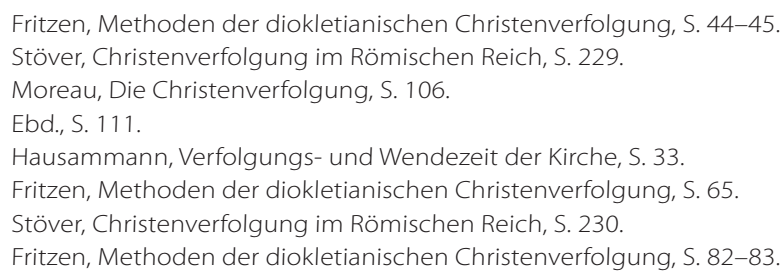


trug, wurde von den Christ_innen als endgültiger Befreier gefeiert. ${ }^{78}$ Er und Konstantin trafen 313 die Mailänder Vereinbarung, die den Christ_innen Religionsfreiheit zusicherte und sämtliche Güter zurückerstattete - ein Edikt hat es nie gegeben. Während sein Name heute fast gänzlich verblasst ist, kennt man Konstantin als den endgültigen Friedensbringer - als es nämlich zwischen den beiden im Jahre 324 zu einem Konflikt kam, ließ Licinius noch römische Gottheiten anbeten, während Konstantin mit christlichen Bannern in die Schlacht zog. Das Ende der Tetrarchie war besiegelt: Konstantin trug den Sieg davon und wurde als Alleinherrscher anerkannt ${ }^{79}$ - dem Ausspruch, dass Geschichte eine Geschichte der Gewinner sei, haftet ein Funken Wahrheit an.

\section{Fazit}

In der Rückschau auf die Ereignisse lässt sich feststellen, dass die große Mehrzahl der Verfolgungen gegen die Christ_innen nicht von staatlicher Seite gelenkt war. Die Beurteilung der Kaiser nach den Kategorien "Verfolger" und "Nicht-Verfolger" lässt sich schon deshalb nicht halten, weil es sich in den meisten Fällen um einzelne Statthalter und Präfekten handelte, die gezielte Verfolgungen initiierten, oder um Pogrome, die von der Bevölkerung ausgingen. Die Christenverfolgungen können deshalb als primär dezentrale Phänomene bezeichnet werden. Eine große Zäsur stellte in dieser Hinsicht der von Decius angeordnete reichsweite Opferbefehl dar. Damit läutete er eine ungefähr siebzigjährige Epoche ein, in der es bei vielen Kaisern eine eindeutige Tendenz gab, Entscheidungen bezüglich der Christ_innen zentral zu treffen. Das Imperium Romanum wollte sich angesichts der seit dem zweiten Jahrhundert von allen Seiten ins Kerngebiet des Reichs eindringenden Stämme der Loyalität seiner Bewohner_innen versichern.

Aus heutiger Sicht ist es problematisch, dass das Thema der Christenverfolgung im Imperium Romanum von der Forschung bisher unverhältnismäßig ausführlich behandelt und immer wieder die Opferrolle hervorgehoben wurde, während die von den Christ_innen betriebenen Verfolgungen heruntergespielt oder marginalisiert wurden.

"Die Zahl aller christlichen Märtyrer in den ersten drei Jahrhunderten wurde auf 1.500 geschätzt, eine wohl sehr problematische Zahl. Erhalten blieb jedenfalls eine schriftliche Kunde überhaupt nur von ein paar Dutzend Märtyrern. Auch sei schon hier daran erinnert, wie gering die damaligen Leiden der Christen erscheinen im Vergleich mit den mittelalterlichen Ketzer- und Hexenverfolgungen. Allein der katholische Herzog Alba ließ mehr als 20.000 Protestanten hinrichten. Die Juden wurden von den Christen zu Hunderttausenden getötet. Die Opfer des kirchlichen Hexenwahnes veranschlagt man auf neun Millionen." ${ }^{\prime 80}$

Moreau resümiert den Geist der Verfolgungen folgendermaßen: 
„Wehe denen - und das gilt bis auf unsere Tage -, die im Krieg oder in Zeiten politischer Krisen nicht das von den jeweiligen Machthabern vorgeschriebene Leben führen!"81

\section{Literatur}

Berchem, Denis van, Tertullians „De pallio“ und der Konflikt des Christentums mit dem Imperium Romanum, in: Richard Klein (Hrsg.), Das frühe Christentum im römischen Staat (Wege der Forschung 267), Darmstadt 1971, S. 106-128.

Borleffs, Jan Willem Philip, Institutum Neronianum, in: Richard Klein (Hrsg.), Das frühe Christentum im römischen Staat (Wege der Forschung 267), Darmstadt 1971, S. 217-235.

Daugherty, Gregory Neil, The cohortes vigilium and the Great Fire of 64 AD, in: Classical Journal 87 (1991), S. 229-240.

Deschner, Karlheinz, Abermals krähte der Hahn. Eine Demaskierung des Christentums von den Evangelisten bis zu den Faschisten, Reinbek 1972.

Ehusani, George, Zum Beispiel Nigeria. Christenverfolgung und soziale Probleme, in: Ursula Spuler-Stegemann (Hrsg.), Feindbild Christentum im Islam. Eine Bestandsaufnahme (Herder Spektrum 5437), Freiburg im Breisgau 2004, S. 129-139.

Flach, Dieter, Die römischen Christenverfolgungen. Gründe und Hintergründe, in: Historia 48 (1999), Heft 4, S. 442-464.

Freudenberger, Rudolf, Das Verhalten der römischen Behörden gegen die Christ_innen im 2. Jahrhundert dargestellt am Brief des Plinius an Trajan und den Reskripten Trajans und Hadrians (Münchener Beiträge zur Papyrusforschung und Antiken Rechtsgeschichte 52), München 1967.

Fritzen, Hedwig, Methoden der diokletianischen Christenverfolgung. Nach der Schrift des Eusebius über die Märtyrer in Palästina, phil. Diss. München 1962.

Gehler, Michael/Rollinger, Robert, Imperien und Reiche in der Weltgeschichte. Epochenübergreifende und globalhistorische Vergleiche, in: Michael Gehler/Robert Rollinger (Hrsg.), Imperien und Reiche in der Weltgeschichte. Epochenübergreifende und globalhistorische Vergleiche 1, Wiesbaden 2014, S. 1-28.

Hausammann, Susanne, Verfolgungs- und Wendezeit der Kirche. Gemeindeleben in der Zeit der Christenverfolgungen und Konstantinischen Wende (Alte Kirche. Zur Geschichte und Theologie in den ersten vier Jahrhunderten 2), Neukirchen-Vluyn 2001.

Henri Grégoire, Les persécutions dans l'Empire romain, Brüssel 1951.

Hermann, Rainer, Die Türkei auf dem Weg nach Europa. Zur Situation der syrisch-orthodoxen Minderheit, in: Ursula Spuler-Stegemann (Hrsg.), Feindbild Christentum im

81 Moreau, Die Christenverfolgung, S. 91. 
Islam. Eine Bestandsaufnahme (Herder Spektrum 5437), Freiburg im Breisgau 2004, S. $87-101$.

Kennedy, Paul, The Rise and Fall of the Great Powers. Economic Change and Military Conflict from 1500 to 2000, New York 1987.

Koep, Leo, Kaisertum und Christusbekenntnis im Widerspruch, in: Richard Klein (Hrsg.), Das frühe Christentum im römischen Staat (Wege der Forschung 267), Darmstadt 1971, S. 302-336.

Moreau, Jacques, Die Christenverfolgung im Römischen Reich (Aus der Welt der Religion 2), Berlin-New York 1971.

Osterhammel, Jürgen, Europamodelle und imperiale Kontexte, in: Journal of Modern European History 2 (2004), S. 157-182.

Portmann, Werner, Zu den Motiven der Diokletianischen Christenverfolgung, in: Historia 39 (1990), Heft 2, S. 212-248.

Riccardi, Andrea, Salz der Erde, Licht der Welt. Glaubenszeugnis und Christenverfolgungen im 20. Jahrhundert, Freiburg im Breisgau 2002.

Rollinger, Robert/Wiesehöfer, Josef, Kaiser Valerian und Ilu-ibdi von Hamat. Über das Schicksal besiegter Feinde, persische Grausamkeit und die Persistenz altorientalischer Traditionen, in: Heather Baker/Kai Kaniuth/Adelheid Otto (Hrsg.), Stories of long ago. Festschrift für Michael D. Roaf (Alter Orient und Altes Testament 397), München 2012, S. 497-516.

Selinger, Reinhard, Die Religionspolitik des Kaisers Decius. Anatomie einer Christenverfolgung (Europäische Hochschulschriften 3/617), Berlin-Bern u. a. 1994.

Olaf Sild, Das altchristliche Martyrium in Berücksichtigung der rechtlichen Grundlage der Christenverfolgung, Dorpat 1920.

Spuler-Stegemann, Ursula, Zum Beispiel Saudi-Arabien. Der wahhabitische Islam als Staatsreligion, in: Ursula Spuler-Stegemann (Hrsg.), Feindbild Christentum im Islam. Eine Bestandsaufnahme (Herder Spektrum 5437), Freiburg im Breisgau 2004, S. $151-$ 155.

Stöver, Hans Dieter, Christenverfolgung im Römischen Reich. Ihre Hintergründe und Folgen, Düsseldorf-Wien 1982.

Ubl, Hannsjörg, Das Regenwunder im Quadenland, in: Alfred Stirnemann (Hrsg.), 1000 Jahre Ostarrîchi. Seine christliche Vorgeschichte. Mission und Glaube im Austausch zwischen Orient und Okzident (Pro Oriente 19), Innsbruck-Wien 1997, S. 16-22.

Vogt, Joseph, Christenverfolgung (historisch), in: Reallexikon für Antike und Christentum 2, Stuttgart 1954, Sp. 1159-1208.

Williams, Daniel, Die Christ_innen des Nahen Ostens - verfolgt und vergessen (Migration 3), Münster 2016. 
Wlosok, Antonie, Rom und die Christ_innen. Zur Auseinandersetzung zwischen Christentum und römischem Staat, in: Der Altsprachliche Unterricht 13 (1970), Beiheft 1.

Zeiller, Jacques, Institutum Neronianum. Hirngespinst oder Wirklichkeit?, in: Richard Klein (Hrsg.), Das frühe Christentum im römischen Staat (Wege der Forschung 267), Darmstadt 1971, S. 236-243.

\section{Quellen}

Augustinus, Vom Gottesstaat (De civitate Dei). Vollständige Ausgabe in einem Band, übersetzt von Wilhelm Thimme, eingeleitet und kommentiert von Carl Andresen, München 2011

Cassius Dio, Dio's Roman History, Bd. 5, Griechisch-englisch, übersetzt von Earnest Cary, herausgegeben von Thomas Ethelbert Page/William Henry Denham Rouse (Loeb Classical Library 82), London-New York 1917.

Cyprian, Des heiligen Kirchenvaters Caecilius Cyprianus Briefe (Bibliothek der Kirchenväter 60), übersetzt von Julius Baer, München 1928.

Draeger, Anton August (Hrsg.), Die Annalen des Tacitus. Schulausgabe, Bd. 2, Leipzig 1869.

Eusebius, Ausgewählte Schriften Band II: Kirchengeschichte, Lateinisch-deutsch, herausgegeben und übersetzt von Philipp Haeuser (Bibliothek der Kirchenväter, 2. Reihe, Bd. 1), München 1932.

Gebhardt, Otto von (Hrsg.), Ausgewählte Märtyreracten und andere Urkunden aus der Verfolgungszeit der christlichen Kirche, Berlin 1902.

Laktanz, Des Lucius Caelius Firmianus Lactantius Schriften (Bibliothek der Kirchenväter, 1. Reihe, Bd. 36), übersetzt von Aloys Hartl, herausgegeben von Otto Bardenhewer/ Thomas Schermann/Carl Weyman, Kempten-München 1919.

Martyrium Polycarpi, Das Martyrium des Polykarp (Kommentar zu den Apostolischen Vätern 6), übersetzt und kommentiert von Gerd Buschmann, Göttingen 1998.

Minucius Felix, Frühchristliche Apologeten, Bd. 2 (Bibliothek der Kirchenväter, 1. Reihe, Bd. 14), übersetzt von Alfons Müller, herausgegeben von Otto Bardenhewer/Thomas Schermann/Carl Weyman, Kempten-München 1913.

Origenes, Acht Bücher gegen Celsus (Bibliothek der Kirchenväter, 1. Reihe, Bd. 52), herausgegeben und übersetzt von Paul Koetschau, München 1926.

Plinius der Jüngere, Des Plinius Cäcilius Secundus Werke (Bd. 4), Lateinisch-deutsch, herausgegeben und übersetzt von Johann Adam Schäfer, Wien-Triest 1814.

Sillig, Karl Julius (Hrsg.), C. Plini Secundi Naturalis Historiae, Bd. 3, Hamburg-Gotha 1853. Sueton, Leben und Taten der römischen Kaiser (Kaiserviten). Über berühmte Männer, herausgegeben und übersetzt von Werner Krenkel und Adolf Stahr, Köln 2013. 
Tertullian, Tertullians ausgewählte Schriften, Bd. 2 (Bibliothek der Kirchenväter, 1. Reihe, Bd. 24), übersetzt von Heinrich Kellner, herausgegeben von Otto Bardenhewer/Thomas Schermann/Carl Weyman, Kempten-München 1915.

Florian Ambach ist Student der Geschichte an der Universität Innsbruck.

Florian.Ambach@student.uibk.ac.at.

\section{Zitation dieses Beitrages}

Florian Ambach, Christenverfolgungen im Römischen Reich. Elemente eines imperialen Niedergangs, in: historia.scribere 10 (2018), S. 193-210, [http://historia.scribere.at], eingesehen 19.6.2018 (=aktuelles Datum).

(C) Creative Commons Licences 3.0 Österreich unter Wahrung der Urheberrechte der Autorlnnen. 